QUARTERLY OF APPLIED MATHEMATICS

VOLUME LXIII, NUMBER 2

JUNE 2005, PAGES 269-275

S 0033-569X(05)00944-7

Article electronically published on April 7, 2005

\title{
A GEOMETRIC APPROACH TO THE EXTENDED D'ALEMBERT PRINCIPLE OF UDWADIA-KALABA-HEE-CHANG
}

\author{
BY \\ JORGE E. SOLOMIN (Universidad Nacional de La Plata, Departamento de Matemática, CC 172, \\ 1900 La Plata, Argentina) \\ AND
}

MARCELA ZUCCALLI (Universidad Nacional de La Plata, Departamento de Matemática, Calle 50 esq 115, 1900 La Plata, Argentina)

Abstract. The extended D'Alembert Principle introduced by Udwadia, Kalaba, and Hee-Chang (1997) is analyzed in the framework developed by Vershik and Fadeev (1981) and shown to be equivalent to the general version of the Principle of virtual work presented therein.

1. Introduction. The celebrated D'Alembert Principle or Principle of virtual work, inspired from the analogous principle characterizing the equilibrium points of static systems, is considered the fundamental tool for describing the dynamics of systems involving non-holonomic constraints (e.g. [5]).

It is sometimes formulated as follows: the work of the constraining force is zero for all virtual displacement from the real trajectory (e.g. [1]).

Under rather general assumptions, this requirement, along with the constraints, allows for computing the constraining force, and then writing down the explicit equations of motions of the system.

A general geometric version of this principle was presented by A. M. Vershik and L. Fadeev in [7] with no explicit consideration of virtual displacements.

One of the first statements of the D'Alembert Principle explicitly addressed to mechanical systems with constraints involving velocity variables in a nonlinear manner is that of [6]. (See, for instance, [3] for examples of such constraints).

The Extended D'Alembert Principle introduced therein is based on a thorough analysis of the concept of virtual displacements, carried out from a dynamical point of view, yielding a rather new definition of them.

Let us recall that, traditionally, virtual displacements has been the name given to the tangent vectors to the configuration manifold of the system satisfying the constraints [1].

Received April 8, 2003.

2000 Mathematics Subject Classification. Primary 70F25; Secondary 70H45, 70G45.

(C)2005 Brown University 
It is worth noting that, as expected, the virtual displacements as considered in [6] coincide with the classical ones if the constraints, whether holonomic or not, are linear in the velocity variables.

The aim of this paper is to give a geometrical interpretation of such an approach and to show that the Extended D'Alembert Principle of [6] turns out to be equivalent to the version of the Principle of virtual work presented in [7].

As a matter of fact, Vershik-Fadeev's theory will have to be slightly enlarged for dealing with time-dependent constraints, since they are not considered in 7 .

2. Virtual displacements. Throughout this section we recall the analysis of the concept of virtual displacements carried out in [6] and its corresponding extended version of the D'Alembert Principle.

Let us consider a mechanical system having an $n$-dimensional manifold $Q$ as configuration space and constrained by the $m$ restrictions

$$
\phi_{i}(q, \dot{q}, t)=0, \quad i=1, \ldots, m,
$$

with

$$
\phi_{i}: T Q \times R \longrightarrow R
$$

smooth functions such that $k(k \leq n)$ of them are independent at each $(q, \dot{q}, t) \in T Q \times R$.

The concept of virtual displacement is approached in [6] in the following way:

The actual displacement $q(t)$ of the constrained system and its velocity $\dot{q}(t)$ are assumed to be known at any given instant of time $t$. At time $t+d t$ one has:

$$
q(t+d t)=q(t)+(d t) \dot{q}(t)+\frac{(d t)^{2}}{2} \ddot{q}(t)+O\left(d t^{3}\right) .
$$

At that instant of time, for any other possible displacement $\tilde{q}(t)$, that is, a displacement satisfying the constraints (1) and such that $\tilde{q}(t)=q(t)$ and $\dot{\tilde{q}}(t)=\dot{q}(t)$, one has

$$
\tilde{q}(t+d t)=q(t)+(d t) \dot{q}(t)+\frac{(d t)^{2}}{2} \ddot{\tilde{q}}(t)+O\left(d t^{3}\right) .
$$

A virtual displacement $w$ at time $t+d t$ is any displacement from the actual configuration $q(t+d t)$ to a possible configuration $\tilde{q}(t+d t)$. Thus,

$$
w(t+d t)=\tilde{q}(t+d t)-q(t+d t)=\frac{(d t)^{2}}{2} v(t)+O\left(d t^{3}\right)
$$

with $v(t)=(\ddot{\tilde{q}}(t)-\ddot{q}(t))$ satisfying

$$
A(q(t), \dot{q}(t), t) v(t)=0
$$

where $A$ is the matrix with entries $A_{i j}=\frac{\partial \phi_{i}}{\partial \dot{q}^{j}}$.

These $v(t)$ s just represent the virtual displacements from the trajectory $q(t)$ at the instant $t$.

It is shown in [6] that for any virtual displacement $w(t+d t)$ there exists such a $v(t)$ satisfying $A(q(t), \dot{q}(t), t) v(t)=0$ and vice-versa. 
REMARK 1. Even for the case of time-dependent constraints, only their derivatives with respect to $\dot{q}$ appear in the matrix $A$. In fact, a virtual displacement must be "...consistent with the instantaneous constraints" [8]. "The displacement is called virtual to distinguish it from an actual displacement of the system occurring in a time interval $d t$, during which the forces and constraints may be changing." [4]

Henceforth, the virtual displacements as defined in [6] will be called dynamical virtual displacements.

3. The geometrical point of view. In this section, we shall analyze dynamical virtual displacements from a geometrical point of view.

We recall that, when considered in the velocities phase space $T Q$, the trajectory $(q(t), \dot{q}(t))$ of a mechanical system is the lifting to $T Q$ of its trajectory $q(t)$ in the configuration manifold $Q$.

Thus, if a vector field $(q, u, v(q, u), w(q, u))$ on $T Q$ is tangent to a trajectory of a mechanical system, it is necessarily a second order equation, that is, $v(q, u)=u$ (these fields are called special fields in [7]).

Let us notice that, on the one hand, if a vector $(v, w) \in T_{\left(q\left(t_{0}\right), \dot{q}\left(t_{0}\right)\right)} T Q$ is a dynamical virtual displacement from $(q(t), \dot{q}(t))$ at $t_{0}$, then $\left(\dot{q}\left(t_{0}\right), \ddot{q}\left(t_{0}\right)\right)+(v, w)$ is tangent at $\left(q\left(t_{0}\right), \dot{q}\left(t_{0}\right)\right)$ to the lifting $(\tilde{q}(t), \dot{\tilde{q}}(t))$ of some trajectory $\tilde{q}(t)$ satisfying $\left(\tilde{q}\left(t_{0}\right), \dot{\tilde{q}}\left(t_{0}\right)\right)=$ $\left(q\left(t_{0}\right), \dot{q}\left(t_{0}\right)\right)$. Therefore, it must hold that $\dot{q}\left(t_{0}\right)+v=\dot{\tilde{q}}\left(t_{0}\right)=\dot{q}\left(t_{0}\right)$, and so $v=\mathbf{0}$. So, a dynamical virtual displacement must be of the form $(\mathbf{0}, w)$.

On the other hand, if we call $\Sigma_{t}$ the submanifold of $T Q$ made up by the states satisfying the constraints at the instant $t$, that is

$$
\Sigma_{t}:=\left\{(q, \dot{q}) \in T Q \text { s.t. } \phi_{i}(q, \dot{q}, t)=0, \text { for } i=1, \ldots, m\right\},
$$

Eq. (2) implies that dynamical virtual displacements at the instant $t$ must be tangent to $\Sigma_{t}$.

Consequently, in our context, the definition of dynamical virtual displacements can be restated in the following way:

Definition 1. Given a mechanical system whose states at time $t$ are constrained to belong to a submanifold $\Sigma_{t}$ of $T Q$, its dynamical virtual displacements at $(q, \dot{q}) \in \Sigma_{t}$ are the vectors $w \in T_{q} Q$ such that $(\mathbf{0}, w) \in T_{(q, \dot{q})} \Sigma_{t}$.

REMARK 2. The vector $w$ can be considered as an element of $T_{q} Q$ because of the isomorphisms $T_{(q, \dot{q})}(T Q) \simeq T_{q} Q \times T_{\dot{q}}\left(T_{q} Q\right)$ and $T_{\dot{q}}\left(T_{q} Q\right) \simeq T_{q} Q$.

In terms of the functions $\phi_{i}$ defining the constraints, the dynamical virtual displacements can be characterized as follows:

Proposition 1. At a given instant of time $t$, a vector $w \in T_{q} Q$ is a dynamical virtual displacement at $(q, \dot{q}) \in \Sigma_{t}$ if and only if

$$
\left(\phi_{i}(q, \dot{q}, t)\right)_{\dot{q}} d q . w=0, \quad \text { for } i=1, \ldots, m .
$$

Proof. At the instant of time $t$, the vector $w \in T_{q} Q$ is a dynamic virtual displacement at $(q, \dot{q}) \Leftrightarrow(\mathbf{0}, w) \in T_{(q, \dot{q})} \Sigma_{t} \Leftrightarrow\left(\phi_{i}(q, \dot{q}, t)\right)_{\dot{q}} d q \cdot w=0$, for $i=1, \ldots, m$. 
Corollary 1. Let us define $\tilde{d} \phi_{i}:=\frac{\partial \phi_{i}}{\partial q} d q+\frac{\partial \phi_{i}}{\partial \dot{q}} d \dot{q}$. Then, at the instant of time $t$, a vector $w \in T_{q} Q$ is a dynamical virtual displacement at a point $(q, \dot{q})$ satisfying the constraints if and only if at that point

$$
\tilde{d} \phi_{i}(\mathbf{0}, w)=0, \text { for } i=1, \ldots, m .
$$

Proof. If, in a local coordinate patch, the vector $w \in T_{q} Q$ can be written as $w=\sum w^{j} \frac{\partial}{\partial q^{j}}$, when considered as an element of $T_{\dot{q}}\left(T_{q} Q\right)$, it reads $w=\sum w^{j} \frac{\partial}{\partial \dot{q}^{j}}$.

So,

$$
\tilde{d} \phi_{i}(\mathbf{0}, w)=\left(\phi_{i}\right)_{\dot{q}} d q \cdot w
$$

Corollary 2. If the functions $\phi_{i}$ do not depend on $t$, a vector $w \in T_{q} Q$ is a dynamical virtual displacement at a point $(q, \dot{q})$ satisfying the constraints if and only if at that point

$$
d \phi_{i}(\mathbf{0}, w)=0, \text { for } i=1, \ldots, m .
$$

Proof. If $\phi_{i}$ does not depend on $t$, then $\tilde{d} \phi_{i}=d \phi_{i}$.

Corollary 3. The dynamical virtual displacements coincide with the classical virtual displacements in the case of restrictions that are linear in the velocity variables.

Proof. If, in a local patch, $\phi_{i}(q, \dot{q}, t)=\sum_{j} a_{i j}(q, t) \dot{q}^{j}$, a vector $w=\sum_{j} w^{j} \frac{\partial}{\partial q^{j}}$ is a classical virtual displacement if and only if $0=\sum_{j} a_{i j}(q, t) w^{j}$ for $i=1, \ldots, m$. So, since $\sum_{j} a_{i j}(q, t) w^{j}=\sum_{j} a_{i j}(q, t) d \dot{q}^{j}(w)=\left(\phi_{i}\right)_{\dot{q}} d q . w$, we get the corollary.

REMARK 3. It is clear that this coincidence does not occur for restrictions that are non-linear in the velocity variables. In particular, at each $t$, the subspace of $T_{q} Q$ made up by the dynamical virtual displacements at $(q, \dot{q})$ also depends on $\dot{q}$ in this case, except for affine restrictions. Let us note that holonomic constraints are always linear in the velocity variables.

REMARK 4. As pointed out in the previous remark, the space of dynamical virtual displacements at $(q, \dot{q})$ can depend on $\dot{q}$ in some cases. Then, if quasi-velocities were introduced (e.g. [2] or [5]), they should be allowed to depend also on $\dot{q}$ and not only on $q$ as it is classically assumed.

The Extended D'Alembert Principle of [6] can now be restated in the following way:

At each instant of time $t$, the constraining force must yield null work along any dynamical virtual displacement.

Under general assumptions, it is shown in [6] that this property and the condition

$$
d \phi_{i}\left(X+\frac{\partial}{\partial t}\right)=0, \text { for } i=1, \ldots, m,
$$

(or, equivalently,

$$
\tilde{d} \phi_{i}(X)=-\frac{\partial \phi_{i}}{\partial t}, \text { for } i=1, \ldots, m
$$


with $\tilde{d} \phi$ as in Corollary 1), for the vector field $X$ generating the dynamics of the constrained system, i.e., $(q(t), \dot{q}(t))$ satisfying the constraints (1), determine the constraining force.

4. The relation with Vershik-Fadeev's approach. Let us first consider the case of constraints $\phi_{i}$ independent of $t$. (Indeed, time-dependent constraints are not analyzed in [7.)

We recall that the "invariant form" of Lagrangian mechanics introduced in [7] is just the symplectic view of Lagrangian mechanics: if $L$ is the Lagrangian function of the system without taking into account the constraints, $T Q$ is endowed with the Lagrangian 2-form $\Omega_{L}=F L^{*} \Omega$, with $\Omega$ the canonical symplectic form on the momentum phase space $T^{*} Q$ and $F L$ the Legendre transform associated to $L$. Forces are represented by horizontal 1-forms on $T Q$, that is, by the 1-forms on $T Q$ vanishing on $(\mathbf{0}, v), \forall v \in T Q$.

REMARK 5. The space of horizontal 1-forms on $T Q$ turns out to be canonically isomorphic to the space of 1 -forms on $Q$ in an obvious way. If $\theta$ is a horizontal 1-form on $T Q$, we shall also denote by $\theta$ the associated 1 -form on $Q$.

In particular, the force represented by $\theta$ yields no work along a vector $v \in T_{q} Q$ if and only if, $\forall w \in T_{q} Q, \theta(v, w)=0$ or, if we consider $\theta$ as a form on $Q, \theta(v)=0$.

The (second order) vector field $X$ on $T Q$ generating the dynamics of the system must satisfy the equations of motion

$$
\Omega_{L}(X, .)=d H_{L}+\beta,
$$

where $H_{L}$ is the energy function corresponding to $L$, that is, $H_{L}(q, v)=F L(v)(v)-$ $L(q, v)$ and $\beta$ represents the total external force. (In our case, $\beta=\varphi+\omega$, with $\varphi$ representing the explicitly known exterior force acting on the system and $\omega$ the constraining force.)

For $i=1, \ldots, m$, we shall denote $\theta_{i}$ the horizontal 1 -forms on $T Q$ given by

$$
\theta_{i}(v, w)=d \phi_{i}(0, v) .
$$

The Principle of virtual work as stated in 7] characterizes the constraining force $\omega$ as follows:

The constraining force $\omega$ is represented by the linear combination of the 1-forms $\theta_{i}$ with coefficients in $C^{\infty}(Q)$ (the Lagrange multipliers) yielding

$$
d \phi_{i}(X)=0, \text { for } i=1, \ldots, m .
$$

Virtual displacements are rather hidden in this version of the Principle of virtual work. In order to uncover them, we observe that the linear space spanned by the horizontal 1-forms $\theta_{i}$, i.e., the space of possible constraining forces, can be characterized as follows:

Proposition 2. Let $\Lambda$ be the space of linear combinations of the 1 -forms $\theta_{i}, i=1, \ldots, m$ and $V$ the space of horizontal 1-forms on $T Q$ vanishing on the vectors $(v, w)$ such that $d \phi_{i}(\mathbf{0}, v)=0, i=1, \ldots, m$.

It holds that

$$
\Lambda=V
$$


Proof. It is easy to see that, $\forall(q, u) \in T Q$, if $\Lambda_{q, u}=\left\{\theta_{(q, u)}\right.$ s.t $\left.\theta \in \Lambda\right\}$ and $V_{q, u}=$ $\left\{\beta_{(q, u)}\right.$ s.t $\left.\beta \in V\right\}$, then $\operatorname{dim}\left(\Lambda_{q, u}\right)=\operatorname{dim}\left(V_{q, u}\right)=k$.

On the other hand, since $\theta_{i}(v, w)=d \phi_{i}(\mathbf{0}, v)$, then $\Lambda_{q, u} \subseteq V_{q, u}$.

Therefore,

$$
\Lambda_{q, u}=V_{q, u},
$$

which implies the proposition.

Now, because of this proposition, Corollary 2, and the isomorphism quoted in Remark 5 , the constraining force must be represented by a 1 -form $\theta$ on $T Q$ such that $\theta(v)=0$ for each dynamical virtual displacement $v$.

Thus, the Vershik-Fadeev's characterization of the constraining force can be restated in the following equivalent way:

The constraining force must yield a null work along any dynamical virtual displacements from the physical trajectory, which, as shown in the previous section, is the characterization given in [6].

As mentioned above, Eq. (6) represents the equations of motion of any system in the general theory developed in [7]. It plays the same role there as the second Newton Law does in [6] for writing down the explicit equations governing the dynamics, once the constraining force is computed.

In this way, we see that the Extended D'Alembert Principle of [6] and the version of the Principle of virtual work introduced by A. M. Vershik and L. Fadeev in [7] are equivalent for time-independent constraints.

For time-dependent constraints, we note that, although not considered in [7], only a few minor modifications are needed for Vershik-Fadeev's theory to encompass them:

i) the form $\omega$ representing the constraining force in Eq. (6), the $\theta_{i}$ s defined by Eq. (7), and the Lagrange multipliers can now depend explicitly on $t$ and, consequently, the equality $\Lambda=V$ of Proposition 2 holds for each fixed $t$;

ii) Eq. (8) must be replaced by Eq. (4);

iii) the space of virtual displacements at each point can depend on $t$, though, as recalled in Remark 1, for defining such displacements, the variable $t$ is kept fixed.

Once these modifications are carried out, the equivalence between the Principles introduced in [6] and [7] follows arguing as before, just by using Corollary 1 instead of Corollary 2.

\section{REFERENCES}

[1] V.I. Arnold, Mathematical Methods of Classical Mechanics (second edition). Graduate Texts in Mathematics 60, Springer Verlag, 1989. MR0997295 (90c:58046)

[2] V.I. Arnold, V.V. Kozlov, A.I. Neishtadt, Mathematical Aspects of Classical and Celestial Mechanics (second edition). Springer Verlag, 1993. MR1292466 (95d:58043b)

[3] P. Appell, Traité de Méchanique Rationelle (troisième édition). Gauthier-Villars, 1911.

[4] H. Goldstein, Ch. Poole, J. Safko, Classical Mechanics (third edition). Addison-Wesley, 2002.

[5] Ju.I Neimark, N.A. Fufaev, Dynamics of Nonholonomic Systems. AMS Translations of Mathematical Monographs, Vol. 33, 1972.

[6] F.E. Udwadia, R.E. Kalaba, E. Hee-Chang, Equations of motion for constrained mechanical systems and the extended D'Alembert principle, Quart. Appl. Math. (LV) 2, 321-331 (1997). MR:1447580 (98f:70016) 
[7] A.M. Vershik, Fadeev L., Lagrangian mechanics in invariant form, Selecta Math. Soviet. (1), 339350 (1981).

[8] E.T. Whittaker, A Treatise on the Analytical Dynamics of Particles and Rigid Bodies. Cambridge University Press, 1904. 